\title{
Coincidental pattern avoidance
}

\author{
Bridget EileEn TenneR*
}

\begin{abstract}
There are several versions of permutation pattern avoidance that have arisen in the literature, and some known examples of two different types of pattern avoidance coinciding. In this paper, we examine barred patterns and vincular patterns. Answering a question of Steingrímsson, we determine when barred pattern avoidance coincides with avoiding a finite set of vincular patterns, and when vincular pattern avoidance coincides with avoiding a finite set of barred patterns. There are 720 barred patterns with this property, each having between 3 and 7 letters, of which at most 2 are barred, and there are 48 vincular patterns with this property, each having between 2 and 4 letters and exactly one bond.
\end{abstract}

Keywords AND PHRASEs: Permutation, pattern, barred pattern, vincular pattern, generalized pattern.

\section{Introduction}

A number of phenomena are equivalent to pattern avoidance; that is, the objects possessing a particular property $P$ can be described by permutations, and these permutations are precisely the permutations that avoid a particular set of patterns $S(P)$. For example, the permutations whose principle order ideals in the Bruhat order are boolean are exactly those permutations that avoid the two patterns 321 and 3412 [11]. For classical pattern avoidance, such phenomena are catalogued in [10].

The classical idea of pattern avoidance began with the work of Simion and Schmidt [8]. Since then, variations on the theme have been developed, including barred patterns, vincular patterns, bi-vincular patterns, mesh patterns, marked mesh patterns, and Bruhat-restricted patterns. The purpose of the current paper is to describe the relationship between the first two of these variations: barred patterns and vincular patterns. Barred patterns were first introduced by West [14], where he showed that two-stack sortable permutations are exactly those that avoid a particular classical pattern on

\footnotetext{
${ }^{*}$ Research partially supported by a DePaul University Competitive Research Leave Grant.
} 
4 letters and a barred pattern on 5 letters. One of the first enumerative studies of barred patterns was by Pudwell in [7]. Vincular patterns, also called "generalized" or "dashed" patterns, were first studied systematically by Babson and Steingrímsson in [1], where many Mahonian permutation statistics were shown to be linear combinations of vincular patterns. Vincular patterns were also surveyed in [9]. (For the sake of completeness, we point out that the other types of patterns mentioned above can be found in $[2,3,13,15]$, respectively. More generally, patterns in both permutations and words have been explored in the text [6].)

Perhaps surprisingly, given their definitions, there are sporadic instances of different types of pattern avoidance coinciding. For example,

$$
\begin{aligned}
\operatorname{Av}(\underline{13} 2) & =\operatorname{Av}(132), \\
\operatorname{Av}(41 \overline{3} 52) & =\operatorname{Av}(3 \underline{14} 2), \quad \text { and } \\
\operatorname{Av}(63 \overline{17} 524) & =\operatorname{Av}(5 \underline{24} 13,63 \underline{15} 24,5 \underline{26} 413)
\end{aligned}
$$

(see Lemma 2.14 and Example 5.2). On the other hand, for any classical permutation $p$, there is no set of barred permutations $\mathrm{B}$ for which $\operatorname{Av}(p)$ and $\operatorname{Av}(B)$ are the same set (see Lemma 2.12).

In this paper, we answer a question of Steingrímsson, posed in [9], to characterize when barred pattern avoidance can be mimicked by avoiding a set of vincular patterns. We also answer the symmetric question, of when vincular pattern avoidance can be mimicked by avoiding a set of barred patterns. These answers appear in Theorem 5.1 and Corollary 5.6, respectively, and it turns out that there are exactly 720 barred patterns that can be mimicked in this way, and 48 vincular patterns that can be. The elements in this latter category are listed explicitly in Table 3.

Section 2 is devoted to carefully defining barred and vincular patterns, giving examples of each, and exploring basic properties. In Sections 3 and 4, we lay the groundwork for stating and proving the main results of the paper, which occur in Sections 5 and 6, respectively. Finally, in the last section, we suggest two directions for further research on this topic.

\section{Flavors of pattern avoidance}

When studying permutation patterns, it is most useful to write permutations in one-line notation, although it should be noted that a relationship between patterns and reduced decompositions was shown in [12]. Throughout this paper, all permutations will be written in one-line notation. 
Definition 2.1. For a positive integer $n$, the set of integers $\{1, \ldots, n\}$ is denoted $[n]$.

Example 2.2. The permutation 52134 is the automorphism of [5] defined by $1 \mapsto 5,2 \mapsto 2,3 \mapsto 1,4 \mapsto 3$, and $5 \mapsto 4$.

The classical concept of pattern avoidance is that a permutation $w$ contains a pattern $p$ if there is a subsequence of the one-line notation of $w$ that is in the same relative order as the letters in $p$. If $w$ does not contain $p$, then $w$ avoids $p$.

\section{Example 2.3.}

- The permutation 52134 contains the pattern 312 because the letters in the subsequence 513 are in the same relative order as the letters in the pattern 312. There are also three other occurrences of the pattern 312 in 52134: 514, 523, and 524 .

- The permutation 52134 avoids the pattern 1234 because it does not have an increasing subsequence of length 4 .

Patterns, whether they be classical, barred, or vincular, are concerned with the relative order of values in a permutation. Thus, throughout this paper, order isomorphic permutations of subsets of $\mathbb{R}$ will be considered equivalent. This equivalence will be denoted " $\approx$," or "=" when no confusion can arise.

Example 2.4. As permutations of subsets of $\mathbb{R}$, we have $312 \approx \sqrt{5}-10$.

The two pattern generalizations that we study in this work are barred patterns and vincular patterns. Although this paper examines multiple types of patterns simultaneously, it will be obvious from the notation whether a given pattern is classical (no decoration), barred (bars over some symbols), or vincular (brackets beneath some symbols). Given a barred or vincular pattern $p$, the classical pattern underlying $p$, obtained by ignoring all decorations, will be denoted $\|p\|$.

Definition 2.5. A barred pattern is a permutation in which a subset of the letters have bars written over them. A barred pattern is proper if bars cover a proper subset of its letters. A permutation $w$ contains a barred pattern $\mathbf{b}$ if $w$ contains an occurrence of the unbarred portion of $\mathbf{b}$ that is not also part of an occurrence of an entire $\|b\|$-pattern. Otherwise - that is, if each occurrence of the unbarred portion of $\mathbf{b}$ in $w$ is part of $\mathbf{a}\|\mathbf{b}\|$-pattern - the permutation $w$ avoids $\mathbf{b}$. 
Henceforth, all barred patterns will be assumed to be proper and simply called "barred."

Example 2.6. Consider the barred pattern $b=3 \overline{2} 4 \overline{1}$, where the unbarred portion is $34 \approx 12$. For a permutation $w$ to contain $\mathrm{b}$, some 12 -pattern in $w$ must not be part of a 3241-pattern in $w$. The permutation 42351 contains $\mathrm{b}$ because the increasing subsequence 23 is not part of any 3241-pattern. On the other hand, the permutation 43251 avoids $b$.

Generalizing the idea of classical patterns in a different direction, vincular patterns allow some letters in the pattern to be forced to be consecutive, or "bonded" together (hence the terminology).

Definition 2.7. A vincular pattern is a classical permutation in which consecutive symbols, including the left and right endpoints (each of which is denoted “*”), may be bonded together. A vincular pattern is proper if there is at least one bond and if the non-* symbols are not all bonded together. A permutation $w$ contains a vincular pattern $v$ if $w$ contains a $\|v\|$-pattern in which any substring bonded together in $v$ must appear consecutively in $w$. If the left (respectively, right) " $*$ " is bonded to its adjacent symbol in $v$, then the corresponding letter in $w$ must appear in the leftmost (respectively, rightmost) position of $w$. Otherwise, the permutation $w$ avoids $v$.

Henceforth, all vincular patterns will be assumed to be proper, and simply called "vincular."

In a vincular pattern, brackets are drawn to indicate the bonds.

\section{Example 2.8.}

- Consider the vincular pattern $v=3 \underline{14} 2$. The permutation 41532 contains $v$ in two ways: 4153 and 4152 . The permutation 41352 avoids v because, although 4152 is a $\|\mathrm{v}\|$-pattern in 41352, the 1 and the 5 are not consecutive.

- Consider the vincular pattern $\mathrm{v}=* 3 \underline{14} 2$. The permutation 41532 contains $v$ in two ways: 4153 and 4152 . The permutation 24153 avoids $\mathrm{v}$ because the single occurrence of $\|\mathrm{v}\|$ in $w$ does not begin in the leftmost position of 24153 .

In general, we define the set of permutations avoiding a pattern as follows.

Definition 2.9. For a (classical, barred, or vincular) pattern $p$, let $\operatorname{Av}(p)$ be the set of permutations that avoid $p$. Similarly, if $P$ is a collection of 
patterns (possibly of different types), then $\operatorname{Av}(P)$ is the set of permutations simultaneously avoiding all patterns in $P$ :

$$
\operatorname{Av}(P)=\bigcap_{p \in P} \operatorname{Av}(p) .
$$

It will be helpful to note that for any $p \in P$,

$$
\operatorname{Av}(P) \subseteq \operatorname{Av}(p) .
$$

The following lemma follows immediately from Definitions 2.5 and 2.7.

Lemma 2.10. Let $\mathrm{b}$ be a barred pattern and $\vee$ a vincular pattern. Then $\|\mathrm{b}\| \in \mathrm{Av}(\mathrm{b})$ and $\|\mathrm{v}\| \notin \operatorname{Av}(\mathrm{v})$.

Barred and vincular patterns have arisen in numerous contexts in the literature. We highlight a few of these here. Precise definitions of the object they characterize are not relevant to this work, and the interested reader is referred to the citations given.

\section{Example 2.11.}

- The two-stack sortable permutations are $\operatorname{Av}(2341,3 \overline{5} 241)$ (see [14]).

- Baxter permutations are $\operatorname{Av}(41 \overline{3} 52,25 \overline{3} 14)$ (see [5]).

- The elements of $\operatorname{Av}(3142,2413)$ are in bijection with $\beta(1,0)$-trees (see [4]).

- By definition, alternating permutations are $\operatorname{Av}(123,321, * 12)$ and reverse alternating permutations are $\operatorname{Av}(\underline{123}, \underline{321}, \underline{* 21})$.

There are some situations where vincular pattern avoidance can be equivalently phrased in terms of classical pattern avoidance, such as $\operatorname{Av}(\underline{132})=\operatorname{Av}(132)$. However, the same cannot be said for barred pattern avoidance.

Lemma 2.12. Let $p$ be a permutation. There is no set $\mathrm{B}$ of barred patterns such that $\operatorname{Av}(p)=\operatorname{Av}(\mathrm{B})$.

Proof. Suppose, to the contrary, that there is such a B. Let $b \in B$ have minimally many unbarred letters. If $p$ is a permutation of $[n]$, then $\operatorname{Av}(p)$ contains all permutations of $[k]$ for each $k<n$. Thus $\mathrm{b}$ must have at least $n$ unbarred letters. Because $p \notin \operatorname{Av}(p)$, the unbarred portion of b must actually be a $p$-pattern. But then $\|\mathrm{b}\| \notin \operatorname{Av}(p)=\operatorname{Av}(\mathrm{B}) \subseteq \operatorname{Av}(\mathrm{b})$, contradicting Lemma 2.10. 
On the other hand, as pointed out in [9], there are some situations where avoidance of a barred pattern is equivalent to avoidance of a suitably chosen vincular pattern.

\section{Example 2.13.}

- $\operatorname{Av}(41 \overline{3} 52)=\operatorname{Av}(3142)$.

- $\operatorname{Av}(25 \overline{3} 14)=\operatorname{Av}(2413)$.

- $\operatorname{Av}(21 \overline{3} 54)=\operatorname{Av}(2 \underline{14} 3)$.

The veracity of the three parts of Example 2.13 is easy to show, and we prove one of them here. Proofs of the other two are entirely analogous.

Lemma 2.14. $\operatorname{Av}(41 \overline{3} 52)=\operatorname{Av}(3 \underline{14} 2)$.

Proof. Suppose $w \in \operatorname{Av}(41 \overline{3} 52)$. Thus any occurrence of 3142 in $w$ must have a relative value of 2.5 sitting between the " 1 " and the " 4 " in that pattern. Thus $w$ avoids the vincular pattern $3 \underline{142}$, and so $w \in \operatorname{Av}(3 \underline{14} 2)$. Hence $\operatorname{Av}(41 \overline{3} 52) \subseteq \operatorname{Av}(3 \underline{14} 2)$.

If $w \notin \operatorname{Av}(41 \overline{3} 52)$, then there is a 3142-pattern in $w$ that is not part of any 41352-pattern in $w$. Choose this occurrence of 3142 in $w$ so that the positions of " 1 " and " 4 " are as close together in $w$ as possible. This ensures that no values less than the " 2 ," nor greater than the " 3 ," appear between this " 1 " and "4." Because this 3142-pattern is not part of a 41352-pattern, no values between the " 2 " and the " 3 " appear in any of these positions either. Thus the " 1 " and " 4 " must actually be adjacent, and so we have an occurrence of $3 \underline{14}$. Hence $w \notin \operatorname{Av}(3 \underline{14} 2)$, and so $\operatorname{Av}(41 \overline{3} 52) \supseteq \operatorname{Av}(3 \underline{14} 2)$.

In contrast to Example 2.13, it is not the case that avoidance of a barred pattern is always equivalent to avoidance of a finite set of vincular patterns.

Lemma 2.15. There is no finite set of vincular patterns $\mathrm{V}$ for which $\operatorname{Av}(1 \overline{2} 3)=\operatorname{Av}(\mathrm{V})$.

Proof. Suppose, to the contrary, that there is such a finite set $\mathrm{V}$.

Because $1,123 \in \operatorname{Av}(1 \overline{2} 3)$ but $12 \notin \operatorname{Av}(1 \overline{2} 3)$, we must have $* 12 * \in \mathrm{V}$. Moreover, because $123 \in \operatorname{Av}(1 \overline{2} 3)$, this is the only element of $\mathrm{V}$ whose underlying classical pattern is 12 .

Fix an integer $n>0$ and suppose, inductively, that for all $0 \leq k<n$, there is a unique $\mathrm{v}_{\mathrm{k}} \in \mathrm{V}$ with $\left\|\mathrm{v}_{\mathrm{k}}\right\|=k(k-1) \cdots 4312$, and that in fact $\mathrm{v}_{\mathrm{k}}=* k(k-1) \cdots 4312 *$.

Consider $u=n(n-1) \cdots 4312$. Because $u \notin \operatorname{Av}(1 \overline{2} 3)$, it must contain some $v \in \mathrm{V}$. Because $\operatorname{Av}(1 \overline{2} 3)=\operatorname{Av}(\mathrm{V})$, Lemma 2.10 implies $\vee \notin \operatorname{Av}(1 \overline{2} 3)$. 
Thus $\|\mathrm{v}\|=\left\|\mathrm{v}_{\mathrm{k}}\right\|$, for some $k \leq n$. The inductive hypothesis implies that $k=n$, so $\|\mathrm{v}\|=u$ itself. It remains only to determine the bonds in $\mathrm{v}$.

For any $i \in[n-2]$, inserting $(-1) 0$ immediately before the $i$ th letter in $u$ yields an element of $\operatorname{Av}(1 \overline{2} 3)$. This ensures that the $(i-1)$ st and $i$ th letters must be bonded in $v$ (where the 9 th letter is the left endpoint). Moreover,

$$
n(n-1) \cdots 4312(n+1)(n+2) \in \operatorname{Av}(1 \overline{2} 3),
$$

so there must also be a bond between the rightmost letter in $v$ and the right endpoint. It now remains to determine whether 1 is bonded to either of its neighbors in $v$. If at least one of these bonds does not exist, then $v$ would be contained in

$$
(n+1) n \cdots 54123 \in \operatorname{Av}(1 \overline{2} 3) .
$$

Thus 1 must be bonded to both of its neighbors, and so $\mathrm{v}=$ $* n(n-1) \cdots 4312 *$. Therefore, by induction, $* n(n-1) \cdots 4312 * \in \mathrm{V}$ for all $n \geq 0$, contradicting the assumption that $\mathrm{V}$ was a finite set.

In order to classify Lemmas 2.14 and 2.15, we introduce the following terminology.

Definition 2.16. Suppose that $B$ is a finite set of barred patterns and that $\mathrm{V}$ is a finite set of vincular patterns. If $\mathrm{Av}(\mathrm{B})=\mathrm{Av}(\mathrm{V})$, then $\mathrm{B}$ and $\mathrm{V}$ are coincident. If $\mathrm{B}$ (respectively, $\mathrm{V}$ ) is a finite set of barred (respectively, vincular) patterns for which such a set $\mathrm{V}$ (respectively, $\mathrm{B}$ ) exists, then $\mathrm{B}$ (respectively, V) is coincidental. If B (respectively, V) is a coincidental set containing just a single element, then that pattern itself is coincidental.

In [9], Steingrímsson posed the problem of classifying all coincidental barred patterns.

Example 2.17. The barred patterns $41 \overline{3} 52,25 \overline{3} 14$, and $21 \overline{3} 54$ are each coincidental. The vincular patterns $3 \underline{14} 2,2 \underline{41} 3$, and $2 \underline{14} 3$ are each coincidental. The sets $\{41 \overline{3} 52\}$ and $\{3142\}$ are coincident. The barred pattern $1 \overline{2} 3$ is not coincidental.

In the present work, we will refine the notion of a coincidental barred permutation to what we call "naturally coincidental," or "nat-co." This concept will be made precise in Definition 4.4, and is intended to capture the particular prohibitions of the particular barred pattern. This restriction is necessary because of the particularly sensitive nature of barred pattern avoidance. More precisely, to avoid a barred pattern $b$, it is necessary that a pattern property be true for every occurrence of the unbarred portion 
of $b$, and these cannot be systematically ruled out if we are not looking at nat-co barred patterns. Note that it is rare for barred pattern avoidance to characterize a certain phenomenon, and so it is perhaps not surprising that we handle these patterns delicately.

Of the two characterizations, it is optimal to start with coincidental vincular patterns because, as shown in Proposition 3.4, each such pattern is coincident with a single (and, as it turns out, naturally coincidental) barred pattern. Thus both characterizations can be addressed by examining naturally coincidental barred patterns (Theorem 5.1).

\section{Preparation for coincidental vincular patterns}

Definition 3.1. Fix a vincular pattern v. Consider the set $B_{0}(v)$ of barred patterns obtained by replacing a single bond of $v$ by a barred symbol. Define $\mathrm{B}(\mathrm{v}) \subseteq \mathrm{B}_{0}(\mathrm{v})$ to be those elements that, when viewed as classical permutations, do not also contain the vincular pattern $v$.

\section{Example 3.2.}

- $\mathrm{B}(3 \underbrace{14} \underbrace{2 *})=\{41 \overline{3} 52,4253 \overline{1}, 3152 \overline{4}, 3142 \overline{5}\}$.

- $\mathrm{B}(3 \underline{142})=\{41 \overline{3} 52,31 \overline{4} 52,31 \overline{5} 42,425 \overline{1} 3,315 \overline{4} 2,314 \overline{5} 2\}$.

Lemma 2.10 and the definition of $B(v)$ immediately imply the following result.

Corollary 3.3. For any vincular pattern $v$,

$$
\{\|\mathrm{b}\|: \mathrm{b} \in \mathrm{B}(\mathrm{v})\} \subseteq(\mathrm{Av}(\mathrm{v}) \cap \mathrm{Av}(\mathrm{B}(\mathrm{v})))
$$

Elements of $B(v)$ are those barred patterns whose bars reflect the vincular nature of $\mathrm{v}$.

Proposition 3.4. If $\mathrm{v}$ is a coincidental vincular pattern, then $|\mathrm{B}(\mathrm{v})|=1$.

Proof. Suppose, to the contrary, that $\mathrm{Av}(\mathrm{v})=\mathrm{Av}(\mathrm{B})$ for some finite set $\mathrm{B}$ of barred patterns, and that there exist distinct $b, b^{\prime} \in B(v)$. By Lemma 2.10, we know that $\|b\| \in A v(b)$ and $\left\|b^{\prime}\right\| \in A v\left(b^{\prime}\right)$. Also, the definition of $B(v)$ gives

$$
\|b\|,\left\|b^{\prime}\right\| \in \operatorname{Av}(v)=\operatorname{Av}(B)
$$

Suppose that $\mathrm{v}$ is a vincular pattern of $n$ letters. Each barred pattern in $B$ must contain at least $n$ unbarred letters, because $\mathrm{Av}(\mathrm{v})=\mathrm{Av}(\mathrm{B})$ contains all permutations of fewer than $n$ letters. The barred patterns $b$ and $b^{\prime}$ each 
have $n$ unbarred letters and a single unbarred letter, meaning that they must be elements of B itself in order to satisfy equation (1). However, because

$$
\|b\| \notin \operatorname{Av}\left(b^{\prime}\right) \supseteq\left(\operatorname{Av}(b) \cap \operatorname{Av}\left(b^{\prime}\right)\right) \supseteq \operatorname{Av}(B)=\operatorname{Av}(v),
$$

this contradicts equation (1).

If $B(v)=0$, then $\operatorname{Av}(v)=\operatorname{Av}(\|v\|)$. But, by Lemma 2.12, such a v would not be coincidental.

Thus $\mathrm{B}(\mathrm{v})=1$.

Suppose that $\mathrm{v}$ is a coincidental vincular pattern. Then, by Proposition 3.4, we have $B(v)=\{b\}$, and $\operatorname{Av}(v)=\operatorname{Av}(b)$. This $b$ has the particular form dictated by the definition of $B(v)$, and it will be clear from Definition 4.4 that this $\mathrm{b}$ is then a naturally coincidental vincular pattern. Thus the problem of determining exactly which vincular patterns are coincidental is a special case of the problem of determining exactly which barred patterns are nat-co, which will be treated in Theorem 5.1.

\section{Preparation for (naturally) coincidental barred patterns}

We now define what it means for a barred pattern to be nat-co.

Definition 4.1. Fix a barred pattern b. Remove a nonempty subset of the barred letters from $b$, and replace each of them by a bond between the newly adjacent letters. Finally, obtain a vincular pattern by ignoring any bars (not the barred letters themselves) that remain. Let $\mathrm{V}(\mathrm{b})$ be the set of vincular patterns obtained in this way.

Some of the vincular patterns obtained by the process described in Definition 4.1 could be equivalent, as in the second example below.

\section{Example 4.2.}

- $\mathrm{V}(4 \overline{13} 5 \overline{2})=\{\underline{32} 41,3 \underline{14} 2,312 \underline{4} *, 231,2 \underline{13}, \underline{21} \underline{3 *}, \underline{12 *}\}$

- $\mathrm{V}(5 \overline{1234} 6)=\{41235,41235,41235,41235,3124,3124,3124$, $3124,3124,3124,213,213,213,12\}$.

The elements of $\mathrm{V}(\mathrm{b})$ are those vincular patterns whose bonds reflect the barred nature of $\mathrm{b}$. As such, these will be the only vincular patterns we allow in our attempt to have vincular patterns mimic the behavior of a barred pattern.

The next result follows immediately from the definition of the set $\mathrm{V}(\mathrm{b})$, and is a complement to Corollary 3.3. 
Corollary 4.3. For any barred pattern b,

$$
\{\|\mathrm{v}\|: \mathrm{v} \in \mathrm{V}(\mathrm{b})\} \cap \operatorname{Av}(\mathrm{b})=\emptyset .
$$

Definition 4.4. A barred pattern $\mathrm{b}$ is naturally coincidental, or nat-co, if $\operatorname{Av}(b)=\operatorname{Av}(V(b))$.

To show that $1 \overline{2} 3$ is not nat-co, it would suffice to prove that $\operatorname{Av}(1 \overline{2} 3) \neq$ $\operatorname{Av}(12)$. Thus Lemma 2.15 actually proves a stronger result about the barred pattern $1 \overline{2} 3$.

In order to characterize nat-co barred patterns, we will need to consider their maximal factors (that is, consecutive subsequences) of barred letters, together with the letters (if any) that appear immediately to the left and to the right of these factors. To this end, we define "boycotts," so-named to refer to the collective barring of a set of values.

Definition 4.5. Let $\mathrm{b}$ be a barred pattern. Suppose that $\overline{\mathrm{b}_{i} \mathrm{~b}_{i+1} \cdots \mathrm{b}_{j}}$ is a barred factor in $b$, of maximal length. Then $X=\left\{b_{i-1}, b_{i}, \ldots, b_{j}, b_{j+1}\right\}$ is a boycott of b. Let $U(X)=\left\{\mathrm{b}_{i-1}, \mathrm{~b}_{j+1}\right\}$ be the set of unbarred letters in $X$, and $B(X)=\left\{\mathrm{b}_{i}, \ldots, \mathrm{b}_{j}\right\}$ be the set of barred letters in $X$.

Note that either element of the set $U(X)$ may be undefined, although they are not both undefined because we have assumed that all barred patterns are proper barred patterns.

Example 4.6. The barred pattern $\overline{92} 43 \overline{71} 5 \overline{6} 8$ has three boycotts. From left to right, they are $\{2,4,9\},\{1,3,5,7\}$, and $\{5,6,8\}$. Moreover, $U(\{2,4,9\})=$ $\{4\}$ and $B(\{2,4,9\})=\{2,9\}$.

The definition of a boycott gives the following easy result.

Lemma 4.7. Any distinct boycotts in a barred pattern share at most one letter, and that letter is unbarred.

\section{Main results}

This paper gives a complete description of all naturally coincidental barred patterns and all coincidental vincular patterns. These results are stated as Theorem 5.1 and Corollary 5.6, respectively.

Theorem 5.1. A barred pattern of $n$ letters is naturally coincidental if and only if it has a unique boycott $X$ and satisfies

- $|B(X)| \leq 2$, 
- for all distinct $x, x^{\prime} \in X$ with $\left\{x, x^{\prime}\right\} \neq U(X)$, we have $\left|x-x^{\prime}\right|>1$, and

- for all $0 \leq k \leq n$, we have $\{k, k+1\} \cap X \neq \emptyset$.

Note that the last bullet point of Theorem 5.1 means $\{1, n\} \in X$, and all three requirements force $3 \leq n \leq 7$.

The proof of this result will follow from a sequence of smaller results, and is presented in Section 6.

Example 5.2. It is easy to check that $1 \overline{4} 23, \overline{15} 324$, and $63 \overline{17} 524$ each satisfy the hypotheses of Theorem 5.1. The conclusion for each case is given below.

- $\operatorname{Av}(1 \overline{4} 23)=\operatorname{Av}(\underline{12} 3)$.

- $\operatorname{Av}(\overline{15} 324)=\operatorname{Av}(* 213,1324, * 4213)$.

- $\operatorname{Av}(63 \overline{17} 524)=\operatorname{Av}(52413,63 \underline{15} 24,526413)$.

It is interesting to note that the non-boycott portion of a nat-co barred pattern is unspecified, once the hypotheses of Theorem 5.1 are met. For example, $\overline{15} 324$ and $\overline{15} 342$ are both nat-co barred patterns.

The restrictive nature of Theorem 5.1 yields several easy corollaries.

Corollary 5.3. A barred pattern of $n$ letters is nat-co if and only if it as a unique boycott $X$ and satisfies

- $n=7$ and $X=\{1,3,5,7\}$, with $|B(X)|=2$,

- $n=6$ and $(U(X), B(X))$ is one of the following pairs:

$$
(\{1,2\},\{4,6\}),(\{3,4\},\{1,6\}), \quad \text { or } \quad(\{5,6\},\{1,3\}),
$$

- $n=5$ and $X=\{1,3,5\}$, with $|B(X)| \in\{1,2\}$,

- $n=4$ and $(U(X), B(X))$ is one of the following pairs:

$$
(\{1,2\},\{4\}) \text { or }(\{3,4\},\{1\}), \quad \text { or }
$$

- $n=3$ and $X=\{1,3\}$, with $|B(X)|$ necessarily equal to 1 .

Note that Corollary 5.3 points out that for a nat-co barred pattern $b$, there will be no degeneracy in the set $\mathrm{V}(\mathrm{b})$ as there had been in the second part of Example 4.2.

Corollary 5.4. For any nat-co barred pattern $\mathrm{b}$, the size of the set $\mathrm{V}(\mathrm{b})$ is either 1 or 3 . More precisely, if $\mathrm{b}$ has a single barred symbol then $|\mathrm{V}(\mathrm{b})|=1$, and if $\mathrm{b}$ has two barred symbols then $|\mathrm{V}(\mathrm{b})|=3$.

Corollary 5.5. There are 720 nat-co barred patterns. 
Table 1: The number of nat-co barred patterns having $n$ letters, of which $b$ are barred. There are 0 nat-co barred patterns for each $(n, b)$ not listed in the table

\begin{tabular}{r||c|c|c|c|c} 
& $n=3$ & 4 & 5 & 6 & 7 \\
\hline \hline$b=1$ & 4 & 8 & 36 & 0 & 0 \\
\hline 2 & 0 & 0 & 24 & 72 & 576
\end{tabular}

Table 2: Examples of nat-co barred patterns having $n$ letters, of which $b$ are barred

\begin{tabular}{r||c|c|c|c|c} 
& $n=3$ & 4 & 5 & 6 & 7 \\
\hline \hline$b=1$ & $\overline{3} 12$ & $1 \overline{4} 23$ & $1 \overline{5} 324$ & - & - \\
\hline 2 & - & - & $\overline{35} 124$ & $1 \overline{46} 235$ & $1 \overline{573246}$
\end{tabular}

Corollary 5.5 is refined in Table 1 .

An example of a nat-co barred permutation for each of the nonzero entries in Table 1 is given in Table 2.

The complementary result to Theorem 5.1, the characterization of coincidental vincular patterns, is now very easy to state. It follows from Proposition 3.4, and Definitions 3.1 and 4.1, that a vincular pattern $v$ is coincidental if and only if $B(v)=\{b\}$ for some nat-co barred pattern $b$.

Corollary 5.6. There are 48 coincidental vincular patterns, all of which were yielded by Theorem 5.1.

The 48 patterns of Corollary 5.6 are listed in Table 3. Note that the first entry in each of the three columns of Table 3 has its corresponding coincidental barred pattern in the $b=1$ row of Table 2 .

\section{Proof of Theorem 5.1}

The proof of Theorem 5.1 will be given in several steps. We first show that each boycott in a nat-co barred pattern must contain both the smallest and largest letters in the pattern, and hence there is exactly one boycott. We will then describe the minimal and maximal distance between values in the boycott, finally concluding that there are at most two barred letters in the boycott.

Proposition 6.1. Let $\mathrm{b}$ be a nat-co barred pattern of $n$ letters and $X$ a boycott in $\mathrm{b}$. Then $1, n \in X$.

Proof. Suppose, to the contrary, that $1 \notin X$. (The proof that $n \in X$ is entirely analogous.) 
Table 3: All coincidental vincular patterns, organized by number of letters, $n$, in the pattern

\begin{tabular}{|c|c|c|c|c|c|}
\hline$n=2$ & $n=3$ & & $n=4$ & & \\
\hline$* 12$ & 123 & 312 & 1324 & 2134 & $24 \underline{13}$ \\
\hline$* 21$ & 213 & 321 & 1342 & $4 \underline{13} 2$ & $42 \underline{13}$ \\
\hline $12 *$ & 231 & $1 \overline{23}$ & 3124 & 2314 & $24 \overline{31}$ \\
\hline \multirow[t]{9}{*}{$21 *$} & 321 & 132 & 31,42 & 4312 & 4231 \\
\hline & & & 1423 & 2143 & 2314 \\
\hline & & & 1432 & 3142 & $32 \overline{14}$ \\
\hline & & & 4123 & 2413 & 2341 \\
\hline & & & $\overline{41} 32$ & $3 \overline{41} 2$ & $32 \overline{41}$ \\
\hline & & & 2413 & 1243 & 1324 \\
\hline & & & 2431 & 3241 & 3124 \\
\hline & & & 4213 & 1423 & $13 \overline{42}$ \\
\hline & & & 4231 & 3421 & 3142 \\
\hline
\end{tabular}

Write $U(X)=\left\{\mathrm{b}_{i-1}, \mathrm{~b}_{j+1}\right\}$ and $B(X)=\left\{\mathrm{b}_{i}, \mathrm{~b}_{i+1}, \ldots, \mathrm{b}_{j}\right\}$, where one or both of the elements of $U(X)$ may be undefined. Let $v \in \mathrm{V}(\mathrm{b})$ be the unique vincular pattern in $V(b)$ having fewest letters, obtained from $b$ by replacing all of its barred symbols by bonds. Let $z$ be the classical permutation obtained by inserting a new smallest symbol between the bonded letters $\mathrm{b}_{i-1}$ and $b_{j+1}$ in $v$. Note that

$$
z \notin \operatorname{Av}(\mathrm{b})
$$

so $z$ must contain some element of $\mathrm{V}(\mathrm{b})$.

Because $1 \notin B(X)$, we have that $z \notin\left\{\left\|\mathrm{v}^{\prime}\right\|: \mathrm{v}^{\prime} \in \mathrm{V}(\mathrm{b})\right\}$. Similarly, because $1 \notin U(X)$, the permutation $z$ does not contain the vincular pattern $v$. Because $v$ is the unique element of $\mathrm{V}(\mathrm{b})$ using the fewest letters, and because $z$ has just one more letter than $\mathrm{v}$ does, we see that $z$ does not contain any of the vincular elements of $\mathrm{V}(\mathrm{b})$ as patterns, and so

$$
z \in \operatorname{Av}(\mathrm{V}(\mathrm{b}))
$$

This contradicts the assumption that $\operatorname{Av}(b)=\operatorname{Av}(\mathrm{V}(\mathrm{b}))$.

The following corollary is an immediate result of Lemma 4.7 and Propostion 6.1.

Corollary 6.2. A nat-co barred pattern b has exactly one boycott.

For the remainder of this section, suppose that $\mathrm{b}$ is a nat-co barred pattern of $n$ letters, with unique boycott $X$. Moreover, let $U(X)=\left\{\mathrm{b}_{i-1}, \mathrm{~b}_{j+1}\right\}$ and $B(X)=\left\{\mathrm{b}_{i}, \mathrm{~b}_{i+1}, \ldots, \mathrm{b}_{j}\right\}$.

Consider the proximity of values that appear in $X$. 
Proposition 6.3. For any distinct $x, x^{\prime} \in B(X)$, we have $\left|x-x^{\prime}\right|>1$.

Proof. Suppose, to the contrary, that there exist $i \leq h<h^{\prime} \leq j$ with $\left|\mathrm{b}_{h}-\mathrm{b}_{h^{\prime}}\right|=1$. Then

$$
b_{1} \cdots b_{h-1} \underline{b}_{b_{h^{\prime}+1}}^{b_{h^{\prime}+2}} \cdots b_{n} \in V(b)
$$

is contained in $b$. Thus $b \notin \operatorname{Av}(\mathrm{V}(\mathrm{b}))=\operatorname{Av}(\mathrm{b})$, contradicting Lemma 2.10.

Proposition 6.4. For any $x \in B(X)$ and $x^{\prime} \in U(X)$, we have $\left|x-x^{\prime}\right|>1$. Proof. Suppose, to the contrary, that there exists $\mathrm{b}_{h} \in B(X)$ such that (without loss of generality) $\left|\mathrm{b}_{h}-\mathrm{b}_{i-1}\right|=1$. Then

$$
b_{1} \cdots b_{i-2} \underbrace{}_{b_{i-1} b_{h+1}} b_{h+2} \cdots b_{n} \in V(b)
$$

is contained in $\mathrm{b}$. Thus $\mathrm{b} \notin \mathrm{Av}(\mathrm{V}(\mathrm{b}))=\operatorname{Av}(\mathrm{b})$, again contradicting Lemma 2.10.

The previous two propositions demonstrate that there is a minimal distance between values in $B(X)$ and between $B(X)$ and $U(X)$ values. The next proposition shows that there is also a maximal distance between values in $X$.

Proposition 6.5. For all $0 \leq k \leq n$, we have $\{k, k+1\} \cap X \neq \emptyset$.

Proof. Suppose, to the contrary, that there is such a $k$ with $k, k+1 \notin X$. Consider

$$
z=\mathrm{b}_{1} \cdots \mathrm{b}_{i-1}(k+.5) \mathrm{b}_{j+1} \cdots \mathrm{b}_{n},
$$

obtained by removing the entire barred factor $\mathrm{b}$ and inserting $k+.5$ in its place. By construction, $z \notin\{\|\mathrm{v}\|: \mathrm{v} \in \mathrm{V}(\mathrm{b})\}$, and $z$ does not contain the vincular pattern $\mathrm{b}_{1} \cdots \mathrm{b}_{i-1} \mathrm{~b}_{j+1} \cdots \mathrm{b}_{n} \in \mathrm{V}(\mathrm{b})$. Thus $z \in \operatorname{Av}(\mathrm{V}(\mathrm{b}))$. However, $z \notin \operatorname{Av}(\mathrm{b})$, yielding a contradiction.

Proposition 6.6. $|B(X)| \leq 2$.

Proof. Suppose, to the contrary, that $|B(X)|>2$; that is, $j-i>1$. Then consider

$$
y=\mathrm{b}_{1} \cdots \mathrm{b}_{i-1} \mathrm{~b}_{i+1}\left(\mathrm{~b}_{i+1}+.5\right) \mathrm{b}_{j+1} \cdots \mathrm{b}_{n} .
$$

By Propositions 6.3-6.5, we have $y \in \operatorname{Av}(\mathrm{V}(\mathrm{b}))$. However, once again we have $y \notin \mathrm{Av}(\mathrm{b})$, yielding a contradiction. 
Propositions 6.3-6.6 now prove one direction of Theorem 5.1: if a barred pattern is nat-co then it must satisfy the listed points. The other direction of the theorem is given by the following proposition.

Proposition 6.7. If a barred pattern $\mathrm{b}$ has one of the forms outlined in Corollary 5.3, then $\mathrm{b}$ is nat-co.

Because the rules are so restrictive, Proposition 6.7 is easy to check by hand and relies entirely on the following observation: inserting a value to break the bond in some $v \in \mathrm{V}(\mathrm{b})$ will always yield a permutation that again contains a pattern from $\mathrm{V}(\mathrm{b})$, or else is equal to $\mathrm{b}$ itself. The proof of Lemma 2.14 demonstrates the procedure.

\section{Further directions}

There are two obvious directions for future research. The first is to characterize all sets of coincidental barred or vincular patterns - not just those of cardinality 1. Certainly there are such sets, as indicated by Example 5.2. Also, although our imposed "natural" coincidence restriction for barred patterns was entirely in keeping with their barred behavior, it could be interesting to try to characterized all coincidental barred patterns after relaxing this rule. Barred pattern containment can be especially fussy, and so it is not clear that completely removing the naturality condition would yield interesting results. However, there may still be some intermediate requirement that would be informative.

\section{References}

[1] E. Babson and E. Steingrímsson (2000). Generalized permutation patterns and a classification of the Mahonian statistics. Sém. Lothar. Combin. B44b 18 pp. MR1758852

[2] M. Bousquet-Mélou, A. Claesson, M. Dukes, and S. Kitaev (2010). $(2+2)$-free posets, ascent sequences and pattern avoiding permutations. J. Combin. Theory Ser. A 117 884-909. MR2652101

[3] P. Brändén and A. Claesson (2011). Mesh patterns and the expansion of permutation statistics as sums of permutation patterns. Electron. J. Combin. 18(2) P5. MR2795782

[4] A. Claesson, S. Kitaev, and E. Steingrímsson (2009). Decompositions and statistics for $\beta(1,0)$-trees and nonseparable permutations. Adv. Appl. Math. 42 313-328. MR2502604 
[5] S. Gire. Arbres, permutations à motifs exclus et cartes planaires: quelques problèmes algorithmique et combinatoire. Ph.D. thesis, Université Bordeaux I, 1993.

[6] S. Kitaev (2011). Patterns in Permutations and Words, Monographs in Theoretical Computer Science. Springer Verlag, Berlin. MR3012380

[7] L. Pudwell (2010). Enumeration schemes for permutations avoiding barred patterns. Electron. J. Combin. 17 R29. MR2595489

[8] R. Simion and F. W. Schmidt (1985). Restricted permutations. European J. Combin. 6 383-406. MR0829358

[9] E. Steingrímsson (2010). Generalized permutation patterns - a short survey. In: Permutation Patterns, London Math. Soc. Lecture Note Ser. Cambridge University Press, Cambridge, pp. 137-152. MR2732827

[10] B. E. Tenner. Database of permutation pattern avoidance, published electronically at http://math.depaul.edu/bridget/patterns.html.

[11] B. E. Tenner (2007). Pattern avoidance and the Bruhat order. J. Combin. Theory, Ser. A 114 888-905. MR2333139

[12] B. E. Tenner (2006). Reduced decompositions and permutation patterns. J. Algebraic Combin. 24 263-284. MR2260018

[13] H. Úlfarsson. A unification of permutation patterns related to Schubert varieties, to appear in Pure Math. Appl. 22 (2011) 273-296. MR2924751

[14] J. West. Permutations with forbidden subsequences and stack-sortable permutations. Ph.D. thesis, MIT, 1990. MR2716312

[15] A. Woo and A. Yong (2006). When is a Schubert variety Gorenstein? Adv. Math. 207 205-220. MR2264071

Bridget EileEn Tenner

Department of Mathematical Sciences

DePaul University

ChicAgo, IL 60614

USA

E-mail address: bridget@math.depaul.edu

URL: http://math.depaul.edu/bridget/

Received March 3, 2013 